\title{
Augmented Lagrangian Method for Total Variation Based Image Restoration and Segmentation Over Triangulated Surfaces
}

\author{
Chunlin Wu • Juyong Zhang • Yuping Duan • \\ Xue-Cheng Tai
}

Received: 31 October 2010 / Revised: 21 February 2011 / Accepted: 28 February 2011

(C) Springer Science+Business Media, LLC 2011

\begin{abstract}
Recently total variation (TV) regularization has been proven very successful in image restoration and segmentation. In image restoration, TV based models offer a good edge preservation property. In image segmentation, TV (or vectorial TV) helps to obtain convex formulations of the problems and thus provides global minimizations. Due to these advantages, TV based models have been extended to image restoration and data segmentation on manifolds. However, TV based restoration and segmentation models are difficult to solve, due to the nonlinearity and non-differentiability of the TV term. Inspired by the success of operator splitting and the augmented Lagrangian method (ALM) in 2D planar image processing, we extend the method to TV and vectorial TV based image restoration and segmentation on triangulated surfaces, which are widely used in computer graphics and computer vision. In particular, we will focus on the following problems. First, several Hilbert spaces will be given to describe TV and vectorial TV based variational models in the discrete setting. Second, we present ALM applied to TV and vectorial TV image restoration on mesh surfaces, leading to efficient algorithms for both gray and color image restoration. Third, we discuss ALM for vectorial TV based multi-region image segmentation, which also works for both gray and color images. The proposed method benefits from fast solvers for
\end{abstract}

C. $\mathrm{Wu}(\bowtie)$

Department of Mathematics, NUS, Block S17, 10, Lower Kent Ridge Road, Singapore 119076,

Singapore

e-mail: wucl@ustc.edu.cn

J. Zhang

School of Computer Engineering, Nanyang Technological University, Singapore, Singapore

e-mail: S070051@e.ntu.edu.sg

Y. Duan · X.-C. Tai

MAS, SPMS, Nanyang Technological University, Singapore, Singapore

Y. Duan

e-mail: DUAN0010@e.ntu.edu.sg

X.-C. Tai

University of Bergen, Bergen, Norway

e-mail: tai@cma.uio.no 
sparse linear systems and closed form solutions to subproblems. Experiments on both gray and color images demonstrate the efficiency of our algorithms.

Keywords Image restoration · Image segmentation · Total variation · Triangulated surfaces · Operator splitting · Augmented Lagrangian method

\section{Introduction}

Since the pioneering work of Rudin et al. [1], TV [1] and vectorial TV models [2-5] have been demonstrated very successful in image restoration. The success of TV and vectorial TV relies on its good edge preserving property, which suits most images where the edges are sparse. For years, these restoration models are usually solved by gradient descent method, which is quite slow due to the non-smoothness of the objective functionals. Recently, various convex optimization techniques have been proposed to efficiently solve these models [6-20], some of which are closely related to iterative shrinkage-thresholding algorithms [21-24].

In addition to image restoration, TV, or vectorial TV, plays an important role in convexifying variational image segmentation models. Although classical variational segmentation models and their gradient descent minimization methods have had a great success, such as snakes [25], geodesic active contours [26, 27], the Chan-Vese method [28] and level set [29] or piecewise constant level set [30] approximation and implementations [31-33] of the Mumford-Shah model [34], they suffer from the local minima problem due to the nonconvexity of the energy functionals. Very recently, various convexification techniques have been proposed to reformulate non-convex segmentation models to convex ones [35-47] and even more general extensions [48, 49], yielding fast and global minimization algorithms. These convex reformulations and extensions are all based on TV or vectorial TV regularizers.

Due to these successes, TV based restoration and segmentation models have been extended to data processing on triangulated manifolds [50-52] via gradient descent method. When this paper was nearly finished, we got to know the technical report [53] released, which is, to the best of our knowledge, the only paper discussing fast convex optimization techniques for these problems. The authors proposed to use split Bregman iteration for TV denoising of gray images and Chan-Vese segmentation (which is a single-phase model). The split Bregman iteration was first introduced in [14] and then was found to be equivalent to the augmented Lagrangian method; see, e.g., $[13,15,17]$ and references therein. In this paper, we would like to present this approach for vectorial TV based image restoration and segmentation problems on triangular mesh surfaces by using the language of augmented Lagrangian method. For the sakes of completeness and readability, we will include the details of both TV and vectorial TV based restorations and segmentations applied to gray and color images, although the former has been discussed in [53] in term of split Bregman iteration. In particular, we will first give several Hilbert spaces to describe TV and vectorial TV based variational models in the discrete setting. Second, ALM applied to TV and vectorial TV image restoration on mesh surfaces will be presented, leading to efficient algorithms for both gray and color image restoration. Third, we discuss ALM for vectorial TV based multi-region (multi-phase) image segmentation, which also works for both gray and color images. For each problem, our algorithms benefit from fast solvers for sparse linear systems and closed form solutions to subproblems.

The paper is organized as follows. In Sect. 2, we introduce some notation. In Sect. 3, we first define several Hilbert spaces with differential mappings, and then present the TV 
and vectorial TV based restoration and segmentation models on triangular mesh surfaces. ALM for TV restoration of gray images, vectorial TV restoration of color images, and multiregion segmentation will be discussed in Sects. 4, 5, 6, respectively. In Sect. 7, we give some numerical experiments. The paper is concluded in Sect. 8 .

\section{Notation}

Assume $M$ is a triangulated surface of arbitrary topology in $\mathbb{R}^{3}$ with no degenerate triangles. The set of vertices, the set of edges, and the set of face triangles of $M$ are denoted as $\left\{v_{i}\right.$ : $\left.i=0,1, \ldots, \mathrm{N}_{v}-1\right\},\left\{e_{i}: i=0,1, \ldots, \mathrm{N}_{e}-1\right\}$, and $\left\{\tau_{i}: i=0,1, \ldots, \mathrm{N}_{\tau}-1\right\}$, respectively, where $\mathrm{N}_{v}, \mathrm{~N}_{e}$, and $\mathrm{N}_{\tau}$ are the numbers of vertices, edges, and triangles. We explicitly denote an edge $e$ whose endpoints are $v_{i}, v_{j}$ as $\left[v_{i}, v_{j}\right]$. Similarly a triangle $\tau$ whose vertices are $v_{i}, v_{j}, v_{k}$ is denoted as $\left[v_{i}, v_{j}, v_{k}\right]$. If $v$ is an endpoint of an edge $e$, then we denote it as $v \prec e$. Similarly, $e$ is an edge of a triangle $\tau$ is denoted as $e \prec \tau$; $v$ is a vertex of a triangle $\tau$ is denoted as $v \prec \tau$ (see [54]). We denote the barycenters of triangle $\tau$, edge $e$, and vertex $v$ by $B C(\tau), B C(e)$, and $B C(v)$, respectively. Let $N_{1}(i)$ be the 1-neighborhood of vertex $v_{i}$. It is the set of indices of vertices that are connected to $v_{i}$. Let $D_{1}(i)$ be the 1-disk of the vertex $v_{i} . D_{1}(i)$ is the set of triangles with $v_{i}$ being one of their vertices. It should be pointed out that the 1-disk of a boundary vertex is topologically just a half disk.

For each vertex $v_{i}$, we define a piecewise linear basis $\phi_{i}$ such that $\phi_{i}\left(v_{j}\right)=\delta_{i j}, i, j=$ $0,1, \ldots, \mathrm{N}_{v}-1$, where $\delta_{i j}$ is the Kronecker delta. $\left\{\phi_{i}: i=0,1, \ldots, \mathrm{N}_{v}-1\right\}$ has the following properties:

1. local support: $\operatorname{supp} \phi_{i}=D_{1}(i)$;

2. nonnegativity: $\phi_{i} \geq 0, i=0,1, \ldots, \mathrm{N}_{v}-1$;

3. partition of unity: $\sum_{0 \leq i \leq \mathrm{N}_{v}-1} \phi_{i} \equiv 1$.

Most data on $M$ are given by assigning values at the vertices of $M$. We can use $\left\{\phi_{i}: i=\right.$ $\left.0,1, \ldots, \mathrm{N}_{v}-1\right\}$ to build piecewise linear functions on $M$ for these data. Suppose $u$ reaches value $u_{i}$ at vertex $v_{i}, i=0,1, \ldots, \mathrm{N}_{v}-1$. Then $u(x)=\sum_{0 \leq i \leq \mathrm{N}_{v}-1} u_{i} \phi_{i}(x)$ for any $x \in M$. Similarly, piecewise linear vector-valued functions $\left(u_{1}(x), u_{2}(x), \ldots, u_{\mathfrak{M}}(x)\right)$ on $M$ can be defined. In some applications, we also have piecewise constant function (vector) over $M$, that is, a single value (vector) is assigned to each triangle of $M$. See [51] and the references therein.

\section{TV Based Restoration and Segmentation Models on Triangulated Surfaces}

In this section, we first define two basic Hilbert spaces on triangular mesh surfaces and differential mappings between them, and then present several TV and vectorial TV based image restoration and segmentation models.

\subsection{Basic Spaces and Differential Mappings}

Given a triangular mesh surface $M$, we now define two Hilbert spaces. The linear space $V_{M}=\mathbb{R}^{\mathrm{N}_{v}}$ is a set, whose elements are given by values at the vertices of $M$. For instance, $u=\left(u_{0}, u_{1}, \ldots, u_{\mathrm{N}_{v}-1}\right) \in V_{M}$ means that $u$ takes value $u_{i}$ at vertex $v_{i}$ for all $i$. By the basis $\left\{\phi_{i}: i=0,1, \ldots, \mathrm{N}_{v}-1\right\}, V_{M}$ is isomorphic to the space of all piecewise linear functions on $M$. We denote, by $Q_{M}$, the set of piecewise constant vector fields on $M$, i.e., $Q_{M}=$ 
$\prod_{\tau \subset M} T \tau$, where $T \tau$ is the tangent vector space of the triangle $\tau$. Note $Q_{M} \subset \mathbb{R}^{3 \times \mathrm{N}_{\tau}}$. For $p \in Q_{M}, p$ restricted in $\tau$ is a constant 3-dimensional vector lying on the tangent space of $\tau$; see [51].

We can equip the spaces $V_{M}$ and $Q_{M}$ with inner products and norms. Motivated by (4.2) in [51], we define, for $u^{1}, u^{2}, u \in V_{M}$,

$$
\left(\left(u^{1}, u^{2}\right)\right)_{V_{M}}=\sum_{0 \leq i \leq \mathrm{N}_{v}-1} u_{i}^{1} u_{i}^{2} s_{i}, \quad\|u\|_{V_{M}}=\sqrt{((u, u))_{V_{M}}}
$$

where $s_{i}=\sum_{\tau \in D_{1}(i)} \frac{1}{3} s_{\tau}$ with $s_{\tau}$ as the area of the triangle $\tau . s_{i}$ is actually the area of the control cell of the vertex $v_{i}$ based on barycentric dual mesh; see [50, 51] and references therein. For $p^{1}, p^{2}, p \in Q_{M}$, we have

$$
\left(\left(p^{1}, p^{2}\right)\right)_{Q_{M}}=\sum_{\tau}\left(p_{\tau}^{1}, p_{\tau}^{2}\right) s_{\tau}, \quad\|p\|_{Q_{M}}=\sqrt{((p, p))_{Q_{M}}},
$$

where $\left(p_{\tau}^{1}, p_{\tau}^{2}\right)$ is the conventional inner product in $\mathbb{R}^{3}$ (with induced norm denoted by $\left|p_{\tau}\right|$ ) and, $s_{\tau}$ is the area of the triangle $\tau$. We mention that, throughout this paper, $(()$,$) and \|\|$ are used to denote inner products and norms of data defined on the whole surface, while $($,$) and$ II are used for those of data restricted on vertices or triangles.

The gradient operator on $M$ is a mapping $\nabla_{M}: V_{M} \rightarrow Q_{M}$. Given $u \in V_{M}$, which corresponds to a piecewise linear function on $M$, its gradient is as follows

$$
\nabla_{M} u=\sum_{0 \leq i \leq \mathrm{N}_{v}-1} u_{i} \nabla_{M} \phi_{i}
$$

where $\nabla_{M} \phi_{i}$ is a piecewise constant vector field on $M$ [51].

Using the above inner products in $V_{M}$ and $Q_{M}$, it is easy to derive the adjoint operator of $-\nabla_{M}$, say, the divergence operator div : $Q_{M} \rightarrow V_{M}$. For $p \in Q_{M}$, div $p$ is given by

$$
(\operatorname{div} p)_{v_{i}}=-\frac{1}{s_{i}} \sum_{\tau, v_{i} \prec \tau} s_{\tau}\left(p_{\tau},\left(\nabla_{M} \phi_{i}\right)_{\tau}\right), \quad \forall i .
$$

Note this is a special case of the divergence formulae in [51] where $X \in Q_{M}$.

\subsection{TV Restoration Model (ROF) on Triangulated Surfaces}

Assume $f \in V_{M}$ is an observed noisy gray (single-valued) image defined on $M$. The total variation restoration model (ROF) aims at solving

$$
\min _{u \in V_{M}}\left\{E_{\mathrm{tv}}(u)=R_{\mathrm{tv}}\left(\nabla_{M} u\right)+\frac{\alpha}{2}\|u-f\|_{V_{M}}^{2}\right\}
$$

where

$$
R_{\mathrm{tv}}\left(\nabla_{M} u\right)=\sum_{\tau}\left|\left(\nabla_{M} u\right)_{\tau}\right| s_{\tau}
$$

is the total variation of $u$, and $\alpha>0$ is a fidelity parameter. Under the assumption of no degenerate triangles on $M$, the objective functional in (5) is coercive, proper, continuous, and strictly convex. Hence, the restoration problem (5) has a unique solution. 


\subsection{Vectorial TV Restoration Model on Triangulated Surfaces}

The TV model (5) can be extended to vectorial TV model for color (multi-valued) image restoration. For the convenience of description, we introduce the following notation (see [17] for similar notation extensions in 2D planar domain):

$$
\begin{aligned}
\mathbf{V}_{M} & =\underbrace{V_{M} \times V_{M} \times \cdots \times V_{M}}_{\mathfrak{M}}, \\
\mathbf{Q}_{M} & =\underbrace{Q_{M} \times Q_{M} \times \cdots \times Q_{M}}_{\mathfrak{M}} .
\end{aligned}
$$

Hence an $\mathfrak{M}$-channel image $\mathbf{u}=\left(u_{1}, u_{2}, \ldots, u_{\mathfrak{M}}\right)$ is an element of $\mathbf{V}_{M}$, and its gradient $\nabla_{M} \mathbf{u}=\left(\nabla_{M} u_{1}, \nabla_{M} u_{2}, \ldots, \nabla_{M} u_{\mathfrak{M}}\right)$ is an element of $\mathbf{Q}_{M}$. The inner products and norms in $\mathbf{V}_{M}$ and $\mathbf{Q}_{M}$ are as follows:

$$
\begin{aligned}
& \left(\left(\mathbf{u}^{1}, \mathbf{u}^{2}\right)\right)_{\mathbf{V}_{M}}=\sum_{1 \leq \mathfrak{m} \leq \mathfrak{M}}\left(\left(u_{\mathfrak{m}}^{1}, u_{\mathfrak{m}}^{2}\right)\right)_{V_{M}}, \quad\|\mathbf{u}\|_{\mathbf{v}_{M}}=\sqrt{((\mathbf{u}, \mathbf{u}))_{\mathbf{V}_{M}}} ; \\
& \left(\left(\mathbf{p}^{1}, \mathbf{p}^{2}\right)\right)_{\mathbf{Q}_{M}}=\sum_{1 \leq \mathfrak{m} \leq \mathfrak{M}}\left(\left(p_{\mathfrak{m}}^{1}, p_{\mathfrak{m}}^{2}\right)\right)_{Q_{M}}, \quad\|\mathbf{p}\|_{\mathbf{Q}_{M}}=\sqrt{((\mathbf{p}, \mathbf{p}))_{\mathbf{Q}_{M}}} .
\end{aligned}
$$

When restricted at a vertex $v_{i}$ (and a triangle $\tau$ ), we can also define the following vertex-byvertex (and triangle-by-triangle) inner products and norms:

$$
\begin{aligned}
\left(\mathbf{u}_{i}^{1}, \mathbf{u}_{i}^{2}\right) & =\sum_{1 \leq \mathfrak{m} \leq \mathfrak{M}}\left(u_{\mathfrak{m}}^{1}\right)_{i}\left(u_{\mathfrak{m}}^{2}\right)_{i}, \quad\left|\mathbf{u}_{i}\right|=\sqrt{\left(\mathbf{u}_{i}, \mathbf{u}_{i}\right)} \\
\left(\mathbf{p}_{\tau}^{1}, \mathbf{p}_{\tau}^{2}\right) & =\sum_{1 \leq \mathfrak{m} \leq \mathfrak{M}}\left(\left(p_{\mathfrak{m}}^{1}\right)_{\tau},\left(p_{\mathfrak{m}}^{2}\right)_{\tau}\right), \quad\left|\mathbf{p}_{\tau}\right|=\sqrt{\left(\mathbf{p}_{\tau}, \mathbf{p}_{\tau}\right)} .
\end{aligned}
$$

We consider the following vector-valued image restoration problem:

$$
\min _{\mathbf{u} \in \mathbf{V}_{M}}\left\{E_{\mathrm{vtv}}(\mathbf{u})=R_{\mathrm{vtv}}\left(\nabla_{M} \mathbf{u}\right)+\frac{\alpha}{2}\|\mathbf{u}-\mathbf{f}\|_{\mathbf{V}_{M}}^{2}\right\},
$$

where

$$
R_{\mathrm{vtv}}\left(\nabla_{M} \mathbf{u}\right)=\mathrm{TV}(\mathbf{u})=\sum_{\tau} \sqrt{\sum_{1 \leq \mathfrak{m} \leq \mathfrak{M}}\left|\left(\nabla_{M} u_{\mathfrak{m}}\right)_{\tau}\right|^{2}} s_{\tau}
$$

is the vectorial TV semi-norm (see [2, 37] for the representation in 2D image domains), and $\mathbf{f}=\left(f_{1}, f_{2}, \ldots, f_{\mathfrak{M}}\right) \in \mathbf{V}_{M}$ is an observed noisy image. Similarly, the restoration problem (6) has a unique minimizer under the assumption of no degenerate triangles on $M$.

Remark More general forms of the restoration models (5) and (6) can be considered, which contain a blur kernel $K$ in the fidelity terms, as in the models in 2D planar image restoration. However, we mention that on general surfaces the blur theory has not been established yet. In non-flat spaces, a blur operator cannot be described as a simple convolution. Algorithms for debluring on surfaces may contain many complicated calculations. 


\subsection{Multi-Region Segmentation on Triangulated Surfaces}

In this sub section, we present the multi-region segmentation model based on vectorial TV regularization. This model has been proposed in $2 \mathrm{D}$ planar image segmentation recently in $[38,42]$ and extended to data segmentation on triangulated manifolds in [52].

We suppose that the $\mathfrak{N}$-channel (either single or multiple channels) data $\mathbf{d}$ on $M$ are to be partitioned to $\mathfrak{M}$ segments. Note that here the number of channels $\mathfrak{N}$ and that of segments $\mathfrak{M}$ are totally independent from each other. In most image data, the segments are assumed to be piecewise constant (for clean data) or approximately piecewise constant (for noisy data). We denote the mean values of the data on these segments as $\mu=\left(\mu_{1}, \ldots, \mu_{\mathfrak{m}}, \ldots, \mu_{\mathfrak{M}}\right)$ (each $\mu_{\mathfrak{m}}$ is an $\mathfrak{N}$-dimensional vector). In this paper we assume $\mu$ is given, since $\mu$ can be estimated in advance in many applications.

In multi-region segmentation, we are to solve

$$
\min _{\mathbf{u} \in C}\left\{E_{\mathrm{mrs}}(\mathbf{u})=((\mathbf{u}, \mathbf{s}))_{\mathbf{V}_{M}}+\beta R_{\mathrm{mrs}}\left(\nabla_{M} \mathbf{u}\right)\right\},
$$

where $R_{\mathrm{mrs}}\left(\nabla_{M} \mathbf{u}\right)=\mathrm{TV}(\mathbf{u}) ; C=\left\{\mathbf{u}=\left(u_{1}, \ldots, u_{\mathfrak{m}}, \ldots, u_{\mathfrak{M}}\right) \in \mathbf{V}_{M}:\left(u_{\mathfrak{m}}\right)_{i} \geq 0\right.$, $\left.\sum_{1 \leq \mathfrak{m} \leq \mathfrak{M}}\left(u_{\mathfrak{m}}\right)_{i}=1, \forall i\right\}$ is the constrained set; $\mathbf{s}=\left(s_{1}, \ldots, s_{\mathfrak{m}}, \ldots, s_{\mathfrak{M}}\right) \in \mathbf{V}_{M}$ and $\left(s_{\mathfrak{m}}\right)_{i}$ indicates the affinity of the data at vertex $v_{i}$ with class $\mathfrak{m} ; \beta$ is a positive parameter. A natural expression of $\left(s_{\mathfrak{m}}\right)_{i}$ is the squared error between the data $\mathbf{d}$ at vertex $v_{i}$ and the mean value $\mu_{\mathfrak{m}}$ :

$$
\left(s_{\mathfrak{m}}\right)_{i}=\left(\mathbf{d}_{i}-\mu_{\mathfrak{m}}, \mathbf{d}_{i}-\mu_{\mathfrak{m}}\right),
$$

which will be considered in this paper. We mention that other affinities with prescribed probability density functions can be similarly treated.

Since $E_{\mathrm{mrs}}(\mathbf{u})$ is continuous and convex and $C$ is convex and closed, the minimization problem (8) has at least one solution.

Remark The model (8) is a convex relaxation [35-49] of the original multi-region segmentation (labeling) problem. The solution is thus not exactly "binary". A "binarization step" is needed to convert the solution of (8) to class numbers. In this paper we use the improved binarization technique proposed in [49].

Remark In the case that the mean values of the segments are unknown and difficult to estimate in advance, we need to solve the following minimization problem:

$$
\min _{\mathbf{u} \in C, \mu}\left\{E_{\mathrm{mrs}}(\mathbf{u}, \mu)=((\mathbf{u}, \mathbf{s}(\mu)))_{\mathbf{V}_{M}}+\beta R_{\mathrm{mrs}}\left(\nabla_{M} \mathbf{u}\right)\right\}
$$

where $R_{\mathrm{mrs}}\left(\nabla_{M} \mathbf{u}\right), C, \beta$ are the same as defined above; and $\mathbf{s}(\mu)$ is also defined by (9) except that here $\mu$ is unknown and thus written as a variable of $\mathbf{s}$. We mention that the problem (10) is non-convex and thus has local minima.

\section{Augmented Lagrangian Method for TV Restoration on Triangulated Surfaces}

Due to the nonlinearity and non-differentiability of the TV model (5), the gradient descent method implemented in [50] is slow. Recently the augmented Lagrangian method has been proven to be very efficient for TV restoration in planar image processing $[15,17,18]$. In the following, we present the method on triangular mesh surfaces. 
We first introduce a new variable $p \in Q_{M}$ and reformulate the problem (5) to be the following equality constrained problem

$$
\begin{aligned}
& \min _{u \in V_{M}, p \in Q_{M}}\left\{G_{\mathrm{tv}}(u, p)=R_{\mathrm{tv}}(p)+\frac{\alpha}{2}\|u-f\|_{V_{M}}^{2}\right\} \\
& \text { s.t. } \quad p=\nabla_{M} u .
\end{aligned}
$$

Problems (5) and (11) are clearly equivalent to each other.

To solve (11), we define the augmented Lagrangian functional

$$
\mathcal{L}_{\mathrm{tv}}(u, p ; \lambda)=R_{\mathrm{tv}}(p)+\frac{\alpha}{2}\|u-f\|_{V_{M}}^{2}+\left(\left(\lambda, p-\nabla_{M} u\right)\right)_{Q_{M}}+\frac{r}{2}\left\|p-\nabla_{M} u\right\|_{Q_{M}}^{2},
$$

with Lagrange multiplier $\lambda \in Q_{M}$ and positive constant $r$, and then consider the following saddle-point problem:

$$
\begin{array}{ll}
\text { Find } & \left(u^{*}, p^{*} ; \lambda^{*}\right) \in V_{M} \times Q_{M} \times Q_{M}, \\
\text { s.t. } & \mathcal{L}_{\mathrm{tv}}\left(u^{*}, p^{*} ; \lambda\right) \leq \mathcal{L}_{\mathrm{tv}}\left(u^{*}, p^{*} ; \lambda^{*}\right) \leq \mathcal{L}_{\mathrm{tv}}\left(u, p ; \lambda^{*}\right), \quad \forall(u, p ; \lambda) \in V_{M} \times Q_{M} \times Q_{M} .
\end{array}
$$

Similarly to [17] and references therein, it can be shown that the saddle-point problem (13) has at least one solution and all the saddle-points $\left(u^{*}, p^{*} ; \lambda^{*}\right)$ 's have the same $u^{*}$, which is the unique solution of the original problem (5).

\section{Algorithm 1 Augmented Lagrangian method for the TV restoration model}

1. Initialization: $\lambda^{0}=0, u^{-1}=0, p^{-1}=0$;

2. For $k=0,1,2, \ldots$ : compute $\left(u^{k}, p^{k}\right)$ as an (approximate) minimizer of the augmented Lagrangian functional with the Lagrange multiplier $\lambda^{k}$, i.e.,

$$
\left(u^{k}, p^{k}\right) \approx \arg \min _{(u, p) \in V_{M} \times Q_{M}} \mathcal{L}_{\mathrm{tv}}\left(u, p ; \lambda^{k}\right),
$$

where $\mathcal{L}_{\mathrm{tv}}\left(u, p ; \lambda^{k}\right)$ is as defined in (12); and update

$$
\lambda^{k+1}=\lambda^{k}+r\left(p^{k}-\nabla_{M} u^{k}\right) .
$$

We now present an iterative algorithm to solve the saddle-point problem. See Algorithm 1 . We are now left with the minimization problem (14) to address. Since $u, p$ are coupled together, it is in general difficult to compute the minimizers $u^{k}$ and $p^{k}$ simultaneously. Instead one usually separates the variables $u$ and $p$ and then applies an alternative minimization procedure $[10,14,15,17]$, through which one can only obtain the minimizer approximately in practice (as an inner iteration of the whole algorithm, this alternative minimization procedure cannot run to infinite steps). See the symbol $\approx$ in $(14)$. Fortunately, we do not need an exact solution to (14) to get the solution to the saddle-point problem.

We separate (14) into the following two subproblems:

- The $u$-sub problem: for a given $p$,

$$
\min _{u \in V_{M}} \frac{\alpha}{2}\|u-f\|_{V_{M}}^{2}-\left(\left(\lambda^{k}, \nabla_{M} u\right)\right)_{Q_{M}}+\frac{r}{2}\left\|p-\nabla_{M} u\right\|_{Q_{M}}^{2} ;
$$


- The $p$-sub problem: for a given $u$,

$$
\min _{p \in Q_{M}} R_{\mathrm{tv}}(p)+\left(\left(\lambda^{k}, p\right)\right)_{Q_{M}}+\frac{r}{2}\left\|p-\nabla_{M} u\right\|_{Q_{M}}^{2} .
$$

These two subproblems can be efficiently solved.

\subsection{Solving the $u$-sub Problem}

The $u$-sub problem (16) is a quadratic programming, whose optimality condition is as follows

$$
\alpha(u-f)+\operatorname{div}_{M} \lambda^{k}+r \operatorname{div}_{M} p-r \operatorname{div}_{M} \nabla_{M} u=0,
$$

which is a sparse linear system and can be solved by various well-developed numerical packages.

\subsection{Solving the $p$-sub Problem}

The $p$-sub problem is decomposable and thus can be solved triangle-by-triangle. For each triangle $\tau$, we need to solve

$$
\min _{p_{\tau}}\left|p_{\tau}\right|+\left(\lambda_{\tau}^{k}, p_{\tau}\right)+\frac{r}{2}\left|p_{\tau}-\left(\nabla_{M} u\right)_{\tau}\right|^{2} .
$$

By a similar geometric argumentation as in [18], (19) has the following closed form solution

$$
p_{\tau}= \begin{cases}\left(1-\frac{1}{r} \frac{1}{\left|w_{\tau}\right|}\right) w_{\tau}, & \left|w_{\tau}\right|>\frac{1}{r}, \\ 0, & \left|w_{\tau}\right| \leq \frac{1}{r},\end{cases}
$$

where

$$
w=\nabla_{M} u-\frac{\lambda^{k}}{r} .
$$

It can be seen that $p_{\tau}$ obtained via (20) is in the tangent vector space $T \tau$ and thus $p \in Q_{M}$, as long as $\lambda_{\tau}^{k} \in T \tau, \forall \tau$, which is a natural consequence from the viewpoint of Hilbert spaces defined previously.

After knowing how to solve the subproblems, we apply an alternative minimization to solve the problem (14). See Algorithm 2. It iteratively and alternatively computes the $u$ and $p$ variables according to (18) and (20). Here $L$ can be chosen using some convergence test techniques. As observed in $[14,15,17]$ and also our numerical experiments, $L=1$ is a good choice and thus applied in this paper.

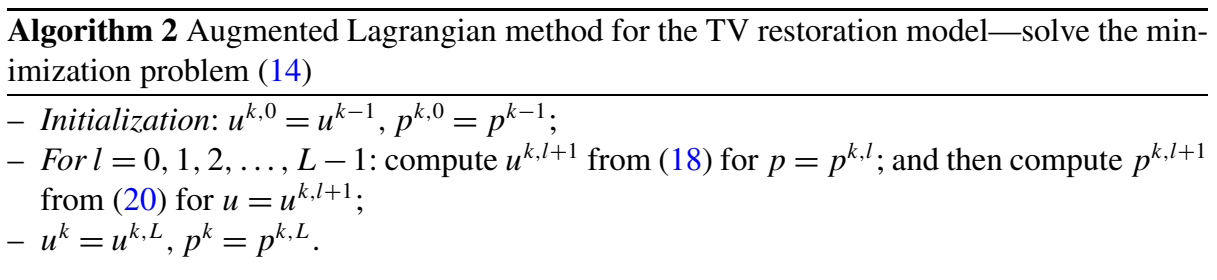

By the zero initialization of Algorithm 1 and induction, it is guaranteed that $u^{k} \in$ $V_{M}, p^{k} \in Q_{M}, \forall k$. 


\section{Augmented Lagrangian Method for Vectorial TV Restoration on Triangulated Surfaces}

The vectorial TV model (6) is also non-differentiable and therefore hard to solve. In this section we present the augmented Lagrangian method for this model, which is extended from the previous section.

By introducing a new variable $\mathbf{p} \in \mathbf{Q}_{M}$, we reformulate the problem (6) to be the following equality constrained problem

$$
\begin{aligned}
& \min _{\substack{\mathbf{u} \in \mathbf{V}_{M}, \mathbf{p} \in \mathbf{Q}_{M} \\
\text { s.t. } \quad \mathbf{p}=\nabla_{M} \mathbf{u} .}}\left\{G_{\mathrm{vtv}}(\mathbf{u}, \mathbf{p})=R_{\mathrm{vtv}}(\mathbf{p})+\frac{\alpha}{2}\|\mathbf{u}-\mathbf{f}\|_{\mathbf{V}_{M}}^{2}\right\} \\
&
\end{aligned}
$$

We then define, for (22), the following augmented Lagrangian functional

$$
\mathcal{L}_{\mathrm{vtv}}(\mathbf{u}, \mathbf{p} ; \lambda)=R_{\mathrm{vtv}}(\mathbf{p})+\frac{\alpha}{2}\|\mathbf{u}-\mathbf{f}\|_{\mathbf{V}_{M}}^{2}+\left(\left(\lambda, \mathbf{p}-\nabla_{M} \mathbf{u}\right)\right)_{\mathbf{Q}_{M}}+\frac{r}{2}\left\|\mathbf{p}-\nabla_{M} \mathbf{u}\right\|_{\mathbf{Q}_{M}}^{2},
$$

with Lagrange multiplier $\lambda \in \mathbf{Q}_{M}$ and positive constant $r$, and consider the saddle-point problem:

Find $\left(\mathbf{u}^{*}, \mathbf{p}^{*} ; \lambda^{*}\right) \in \mathbf{V}_{M} \times \mathbf{Q}_{M} \times \mathbf{Q}_{M}$,

$$
\text { s.t. } \quad \mathcal{L}_{\mathrm{vtv}}\left(\mathbf{u}^{*}, \mathbf{p}^{*} ; \lambda\right) \leq \mathcal{L}_{\mathrm{vtv}}\left(\mathbf{u}^{*}, \mathbf{p}^{*} ; \lambda^{*}\right) \leq \mathcal{L}_{\mathrm{vtv}}\left(\mathbf{u}, \mathbf{p} ; \lambda^{*}\right), \quad \forall(\mathbf{u}, \mathbf{p} ; \lambda) \in \mathbf{V}_{M} \times \mathbf{Q}_{M} \times \mathbf{Q}_{M} .
$$

It can be shown that the saddle-point problem (24) has at least one solution and all the saddle-points $\left(\mathbf{u}^{*}, \mathbf{p}^{*} ; \lambda^{*}\right)$ 's have the same $\mathbf{u}^{*}$, which is the unique solution of the original problem (6).

\section{Algorithm 3 Augmented Lagrangian method for the vectorial TV model}

1. Initialization: $\lambda^{0}=0, \mathbf{u}^{-1}=0, \mathbf{p}^{-1}=0$;

2. For $k=0,1,2, \ldots$ : compute $\left(\mathbf{u}^{k}, \mathbf{p}^{k}\right)$ as an (approximate) minimizer of the augmented Lagrangian functional with the Lagrange multiplier $\lambda^{k}$, i.e.,

$$
\left(\mathbf{u}^{k}, \mathbf{p}^{k}\right) \approx \arg \min _{(\mathbf{u}, \mathbf{p}) \in \mathbf{V}_{M} \times \mathbf{Q}_{M}} \mathcal{L}_{\mathrm{vtv}}\left(\mathbf{u}, \mathbf{p} ; \lambda^{k}\right),
$$

where $\mathcal{L}_{\mathrm{vtv}}\left(\mathbf{u}, \mathbf{p} ; \lambda^{k}\right)$ is as defined in (23); and update

$$
\lambda^{k+1}=\lambda^{k}+r\left(\mathbf{p}^{k}-\nabla_{M} \mathbf{u}^{k}\right)
$$

We use Algorithm 3 to solve the saddle-point problem, where the minimization problem (25) is left to address. Since $\mathbf{u}, \mathbf{p}$ are coupled together in (25), we separate the variables $\mathbf{u}$ and $\mathbf{p}$ and then apply an alternative minimization procedure, as in the method for the TV model (5).

In particular, (25) is separated into the following two subproblems:

- The $\mathbf{u}$-sub problem: for a given $\mathbf{p}$,

$$
\min _{\mathbf{u} \in \mathbf{V}_{M}} \frac{\alpha}{2}\|\mathbf{u}-\mathbf{f}\|_{\mathbf{V}_{M}}^{2}-\left(\left(\lambda^{k}, \nabla_{M} \mathbf{u}\right)\right)_{\mathbf{Q}_{M}}+\frac{r}{2}\left\|\mathbf{p}-\nabla_{M} \mathbf{u}\right\|_{\mathbf{Q}_{M}}^{2}
$$


- The p-sub problem: for a given $\mathbf{u}$,

$$
\min _{\mathbf{p} \in \mathbf{Q}_{M}} R_{\mathrm{vtv}}(\mathbf{p})+\left(\left(\lambda^{k}, \mathbf{p}\right)\right)_{\mathbf{Q}_{M}}+\frac{r}{2}\left\|\mathbf{p}-\nabla_{M} \mathbf{u}\right\|_{\mathbf{Q}_{M}}^{2}
$$

\subsection{Solving the $\mathbf{u}$-sub Problem}

The u-sub problem (27) is a quadratic programming, whose optimality condition is as follows

$$
\alpha(\mathbf{u}-\mathbf{f})+\operatorname{div}_{M} \lambda^{k}+r \operatorname{div}_{M} \mathbf{p}-r \operatorname{div}_{M} \nabla_{M} \mathbf{u}=0,
$$

which is, in channel wise, as follows:

$$
\alpha\left(u_{\mathfrak{m}}-f_{\mathfrak{m}}\right)+\operatorname{div}_{M} \lambda_{\mathfrak{m}}^{k}+r \operatorname{div}_{M} p_{\mathfrak{m}}-r \operatorname{div}_{M} \nabla_{M} u_{\mathfrak{m}}=0, \quad 1 \leq \mathfrak{m} \leq \mathfrak{M} .
$$

Therefore one need only to solve $\mathfrak{M}$ sparse linear systems with the same coefficient matrix. Some well-developed numerical packages can be applied.

\subsection{Solving the $\mathbf{p}$-sub Problem}

The p-sub problem is decomposable and can be solved triangle-by-triangle. For each triangle $\tau$, we need to solve

$$
\min _{\mathbf{p}_{\tau}}\left|\mathbf{p}_{\tau}\right|+\left(\lambda_{\tau}^{k}, \mathbf{p}_{\tau}\right)+\frac{r}{2}\left|\mathbf{p}_{\tau}-\left(\nabla_{M} \mathbf{u}\right)_{\tau}\right|^{2},
$$

which has the following closed form solution

$$
\mathbf{p}_{\tau}= \begin{cases}\left(1-\frac{1}{r} \frac{1}{\left|\mathbf{w}_{\tau}\right|}\right) \mathbf{w}_{\tau}, & \left|\mathbf{w}_{\tau}\right|>\frac{1}{r}, \\ 0, & \left|\mathbf{w}_{\tau}\right| \leq \frac{1}{r},\end{cases}
$$

where

$$
\mathbf{w}=\nabla_{M} \mathbf{u}-\frac{\lambda^{k}}{r} .
$$

It is seen that $\mathbf{p}_{\tau}$ obtained via (32) is in the tangent vector space $T \tau$ and thus $\mathbf{p} \in \mathbf{Q}_{M}$, as long as $\lambda_{\tau}^{k} \in T \tau, \forall \tau$. This is also a natural consequence from the definition of $\mathbf{Q}_{M}$.

We then apply an alternative minimization to solve the problem (25). See Algorithm 4, which iteratively and alternatively computes the $\mathbf{u}$ and $\mathbf{p}$ variables according to (29) and (32). Here $L$ can be chosen using some convergence test techniques. In this paper we simply set $L=1$.

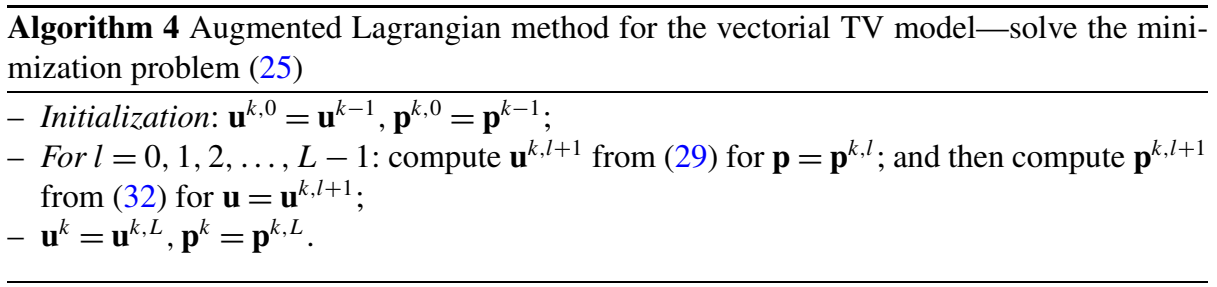

By the zero initialization of Algorithm 3 and induction, it is guaranteed that $\mathbf{u}^{k} \in$ $\mathbf{V}_{M}, \mathbf{p}^{k} \in \mathbf{Q}_{M}, \forall k$. 


\section{Augmented Lagrangian Method for Multi-Region Segmentation on Triangulated Surfaces}

Due to the involvement of the vectorial TV semi-norm, the multi-region segmentation model (8) is non-differentiable and thus hard to solve. In this section we present to use the augmented Lagrangian method to solve it.

By defining

$$
\chi_{C}(\mathbf{u})= \begin{cases}0, & \mathbf{u} \in C, \\ +\infty, & \mathbf{u} \notin C,\end{cases}
$$

the problem (8) can be verified equivalent to the following minimization problem:

$$
\min _{\mathbf{u} \in \mathbf{V}_{M}}\left\{\bar{E}_{\mathrm{mrs}}(\mathbf{u})=((\mathbf{u}, \mathbf{s}))_{\mathbf{V}_{M}}+\beta R_{\mathrm{mrs}}\left(\nabla_{M} \mathbf{u}\right)+\chi_{C}(\mathbf{u})\right\}
$$

where the objective functional $\bar{E}_{\mathrm{mrs}}(\mathbf{u})$ is convex, lower semi-continuous, and proper, as well as coercive over $\mathbf{V}_{M}$, by Cauchy-Schwartz inequality applied to $((\mathbf{u}, \mathbf{s}))_{\mathbf{V}_{M}}$.

The problem (34) can be further reformulated to

$$
\begin{aligned}
& \min _{\mathbf{u} \in \mathbf{V}_{M}, \mathbf{p} \in \mathbf{Q}_{M}, \mathbf{z} \in \mathbf{V}_{M}}\left\{G_{\mathrm{mrs}}(\mathbf{u}, \mathbf{p}, \mathbf{z})=((\mathbf{z}, \mathbf{s}))_{\mathbf{V}_{M}}+\beta R_{\mathrm{mrs}}(\mathbf{p})+\chi_{C}(\mathbf{z})\right\} \\
& \text { s.t. } \quad \mathbf{p}=\nabla_{M} \mathbf{u}, \quad \mathbf{z}=\mathbf{u},
\end{aligned}
$$

by introducing two auxiliary variables $\mathbf{p} \in \mathbf{Q}_{M}$ and $\mathbf{z} \in \mathbf{V}_{M}$. We remark that the introduction of $\mathbf{z}$ avoids a difficult inequality constrained quadratic programming problem (especially for large meshes) and will greatly simplify the calculation.

To solve (35), we define the following augmented Lagrangian functional

$$
\begin{aligned}
\mathcal{L}_{\mathrm{mrs}}\left(\mathbf{u}, \mathbf{p}, \mathbf{z} ; \lambda_{\mathbf{p}}, \lambda_{\mathbf{z}}\right)= & ((\mathbf{z}, \mathbf{s}))_{\mathbf{V}_{M}}+\beta R_{\mathrm{mrs}}(\mathbf{p})+\chi_{C}(\mathbf{z})+\left(\left(\lambda_{\mathbf{p}}, \mathbf{p}-\nabla_{M} \mathbf{u}\right)\right)_{\mathbf{Q}_{M}} \\
& +\left(\left(\lambda_{\mathbf{z}}, \mathbf{z}-\mathbf{u}\right)\right)_{\mathbf{V}_{M}}+\frac{r_{\mathbf{p}}}{2}\left\|\mathbf{p}-\nabla_{M} \mathbf{u}\right\|_{\mathbf{Q}_{M}}^{2}+\frac{r_{\mathbf{z}}}{2}\|\mathbf{z}-\mathbf{u}\|_{\mathbf{V}_{M}}^{2},
\end{aligned}
$$

with Lagrange multipliers $\lambda_{\mathbf{p}} \in \mathbf{Q}_{M}, \lambda_{\mathbf{z}} \in \mathbf{V}_{M}$ and positive constants $r_{\mathbf{p}}, r_{\mathbf{z}}$, and then consider the following saddle-point problem:

$$
\begin{aligned}
& \text { Find }\left(\mathbf{u}^{*}, \mathbf{p}^{*}, \mathbf{z}^{*} ; \lambda_{\mathbf{p}}^{*}, \lambda_{\mathbf{z}}^{*}\right) \in \mathbf{V}_{M} \times \mathbf{Q}_{M} \times \mathbf{V}_{M} \times \mathbf{Q}_{M} \times \mathbf{V}_{M}, \\
& \begin{array}{l}
\text { s.t. } \quad \mathcal{L}_{\mathrm{mrs}}\left(\mathbf{u}^{*}, \mathbf{p}^{*}, \mathbf{z}^{*} ; \lambda_{\mathbf{p}}, \lambda_{\mathbf{z}}\right) \leq \mathcal{L}_{\mathrm{mrs}}\left(\mathbf{u}^{*}, \mathbf{p}^{*}, \mathbf{z}^{*} ; \lambda_{\mathbf{p}}^{*}, \lambda_{\mathbf{z}}^{*}\right) \leq \mathcal{L}_{\mathrm{mrs}}\left(\mathbf{u}, \mathbf{p}, \mathbf{z} ; \lambda_{\mathbf{p}}^{*}, \lambda_{\mathbf{z}}^{*}\right), \\
\quad \forall\left(\mathbf{u}, \mathbf{p}, \mathbf{z} ; \lambda_{\mathbf{p}}, \lambda_{\mathbf{z}}\right) \in \mathbf{V}_{M} \times \mathbf{Q}_{M} \times \mathbf{V}_{M} \times \mathbf{Q}_{M} \times \mathbf{V}_{M} .
\end{array}
\end{aligned}
$$

It can be shown that the above saddle-point problem (37) has at least one solution and each solution $\left(\mathbf{u}^{*}, \mathbf{p}^{*}, \mathbf{z}^{*} ; \lambda_{\mathbf{p}}^{*}, \lambda_{\mathbf{z}}^{*}\right)$ provides a minimizer $\mathbf{u}^{*}$ of the segmentation problem (8).

We now present Algorithm 5 to solve the saddle-point problem (37), where the minimization problem (38) is left to address in later paragraphs. Similarly to those in TV and vectorial TV models, here we have coupled variables $\mathbf{u}, \mathbf{p}, \mathbf{z}$. We need to separate the variables $\mathbf{u}, \mathbf{p}$, and $\mathbf{z}$ and then apply an alternative minimization procedure to solve (38).

In particular, (38) is separated into the following three subproblems:

- The u-sub problem: for a given $\mathbf{p}, \mathbf{z}$,

$$
\min _{\mathbf{u} \in \mathbf{V}_{M}}-\left(\left(\lambda_{\mathbf{p}}^{k}, \nabla_{M} \mathbf{u}\right)\right)_{\mathbf{Q}_{M}}-\left(\left(\lambda_{\mathbf{z}}^{k}, \mathbf{u}\right)\right)_{\mathbf{V}_{M}}+\frac{r_{\mathbf{p}}}{2}\left\|\mathbf{p}-\nabla_{M} \mathbf{u}\right\|_{\mathbf{Q}_{M}}^{2}+\frac{r_{\mathbf{z}}}{2}\|\mathbf{z}-\mathbf{u}\|_{\mathbf{V}_{M}}^{2}
$$


Algorithm 5 Augmented Lagrangian method for multi-region segmentation

1. Initialization: $\lambda_{\mathbf{p}}^{0}=0, \lambda_{\mathbf{z}}^{0}=0, \mathbf{u}^{-1}=0, \mathbf{p}^{-1}=0, \mathbf{z}^{-1}=0$;

2. For $k=0,1,2, \ldots$ : compute $\left(\mathbf{u}^{k}, \mathbf{p}^{k}, \mathbf{z}^{k}\right)$ as an (approximate) minimizer of the augmented Lagrangian functional with the Lagrange multipliers $\lambda_{\mathbf{p}}^{k}, \lambda_{\mathbf{z}}^{k}$, i.e.,

$$
\left(\mathbf{u}^{k}, \mathbf{p}^{k}, \mathbf{z}^{k}\right) \approx \arg \min _{(\mathbf{u}, \mathbf{p}, \mathbf{z}) \in \mathbf{V}_{M} \times \mathbf{Q}_{M} \times \mathbf{V}_{M}} \mathcal{L}_{\mathrm{mrs}}\left(\mathbf{u}, \mathbf{p}, \mathbf{z} ; \lambda_{\mathbf{p}}^{k}, \lambda_{\mathbf{z}}^{k}\right),
$$

where $\mathcal{L}_{\text {mrs }}\left(\mathbf{u}, \mathbf{p}, \mathbf{z} ; \lambda_{\mathbf{p}}^{k}, \lambda_{\mathbf{z}}^{k}\right)$ is as defined in (36); and update

$$
\begin{aligned}
& \lambda_{\mathbf{p}}^{k+1}=\lambda_{\mathbf{p}}^{k}+r_{\mathbf{p}}\left(\mathbf{p}^{k}-\nabla_{M} \mathbf{u}^{k}\right), \\
& \lambda_{\mathbf{z}}^{k+1}=\lambda_{\mathbf{z}}^{k}+r_{\mathbf{z}}\left(\mathbf{z}^{k}-\mathbf{u}^{k}\right) .
\end{aligned}
$$

- The p-sub problem: for a given $\mathbf{u}, \mathbf{z}$,

$$
\min _{\mathbf{p} \in \mathbf{Q}_{M}} \beta R_{\mathrm{mrs}}(\mathbf{p})+\left(\left(\lambda_{\mathbf{p}}^{k}, \mathbf{p}\right)\right)_{\mathbf{Q}_{M}}+\frac{r_{\mathbf{p}}}{2}\left\|\mathbf{p}-\nabla_{M} \mathbf{u}\right\|_{\mathbf{Q}_{M}}^{2}
$$

- The z-sub problem: for a given $\mathbf{u}, \mathbf{p}$,

$$
\min _{\mathbf{z} \in \mathbf{V}_{M}}((\mathbf{z}, \mathbf{s}))_{\mathbf{V}_{M}}+\chi_{C}(\mathbf{z})+\left(\left(\lambda_{\mathbf{z}}^{k}, \mathbf{z}\right)\right)_{\mathbf{V}_{M}}+\frac{r_{\mathbf{z}}}{2}\|\mathbf{z}-\mathbf{u}\|_{\mathbf{V}_{M}}^{2} .
$$

All of these subproblems can be efficiently solved.

\subsection{Solving the $\mathbf{u}$-sub Problem}

The u-sub problem (40) is a quadratic programming, whose optimality condition is as follows

$$
\operatorname{div}_{M} \lambda_{\mathbf{p}}^{k}-\lambda_{\mathbf{z}}^{k}+r_{\mathbf{p}} \operatorname{div}_{M} \mathbf{p}-r_{\mathbf{p}} \operatorname{div}_{M} \nabla_{M} \mathbf{u}-r_{\mathbf{z}}(\mathbf{z}-\mathbf{u})=0,
$$

which is, in channel wise, as follows:

$$
\operatorname{div}_{M}\left(\lambda_{\mathbf{p}}\right)_{\mathfrak{m}}^{k}-\left(\lambda_{\mathbf{z}}\right)_{\mathfrak{m}}^{k}+r_{\mathbf{p}} \operatorname{div}_{M} p_{\mathfrak{m}}-r_{\mathbf{p}} \operatorname{div}_{M} \nabla_{M} u_{\mathfrak{m}}-r_{\mathbf{z}}\left(z_{\mathfrak{m}}-u_{\mathfrak{m}}\right)=0, \quad 1 \leq \mathfrak{m} \leq \mathfrak{M}
$$

These $\mathfrak{M}$ sparse linear systems with the same coefficient matrix can be very efficiently solved by well-developed numerical packages.

\subsection{Solving the $\mathbf{p}$-sub Problem}

The p-sub problem (41) is decomposable and can be solved triangle-by-triangle. For each triangle $\tau$, we need to solve

$$
\min _{\mathbf{p}_{\tau}} \beta\left|\mathbf{p}_{\tau}\right|+\left(\left(\lambda_{\mathbf{p}}\right)_{\tau}^{k}, \mathbf{p}_{\tau}\right)+\frac{r_{\mathbf{p}}}{2}\left|\mathbf{p}_{\tau}-\left(\nabla_{M} \mathbf{u}\right)_{\tau}\right|^{2},
$$

which has the following closed form solution

$$
\mathbf{p}_{\tau}= \begin{cases}\left(1-\frac{\beta}{r_{\mathbf{p}}} \frac{1}{\left|\mathbf{w}_{\tau}\right|}\right) \mathbf{w}_{\tau}, & \left|\mathbf{w}_{\tau}\right|>\frac{\beta}{r_{\mathbf{p}}}, \\ 0, & \left|\mathbf{w}_{\tau}\right| \leq \frac{\beta}{r_{\mathbf{p}}},\end{cases}
$$


where

$$
\mathbf{w}=\nabla_{M} \mathbf{u}-\frac{\lambda_{\mathbf{p}}^{k}}{r_{\mathbf{p}}} .
$$

It can be seen that $\mathbf{p}_{\tau}$ obtained via (46) is in the tangent vector space $T \tau$ and thus $\mathbf{p} \in$ $\mathbf{Q}_{M}$, as long as $\left(\lambda_{\mathbf{p}}\right)_{\tau}^{k} \in T \tau, \forall \tau$. This is also indicated by the Hilbert space in the problem formulation.

\subsection{Solving the $\mathbf{z}$-sub Problem}

The $\mathbf{z}$-sub problem (42) is equivalent to

$$
\min _{\mathbf{z} \in C}((\mathbf{z}, \mathbf{s}))_{\mathbf{V}_{M}}+\left(\left(\lambda_{\mathbf{z}}^{k}, \mathbf{z}\right)\right)_{\mathbf{V}_{M}}+\frac{r_{\mathbf{z}}}{2}\|\mathbf{z}-\mathbf{u}\|_{\mathbf{V}_{M}}^{2},
$$

which is actually a quadratic programming problem where the objective functional is decomposable and the admissible set $C$ is a convex set. Therefore we can first minimize the objective functional over $\mathbf{V}_{M}$ and then project the minimizer to $C$. The solution to (48) reads as follows

$$
\mathbf{z}=\operatorname{Proj}_{C}\left(\mathbf{u}-\frac{\mathbf{s}+\lambda_{\mathbf{z}}^{k}}{r_{\mathbf{z}}}\right),
$$

which can be calculated vertex-by-vertex via Michelot's algorithm [55].

We then apply an alternative minimization to solve the problem (38). See Algorithm 6, which iteratively and alternatively computes the $\mathbf{u}, \mathbf{p}$, and $\mathbf{z}$, according to (43), (46), and (49), respectively. Here $L$ can be chosen using some convergence test techniques. In this paper we simply set $L=1$.

$\overline{\text { Algorithm } 6 \text { Augmented Lagrangian method for multi-region segmentation-solve the }}$ minimization problem (38)

- Initialization: $\mathbf{u}^{k, 0}=\mathbf{u}^{k-1}, \mathbf{p}^{k, 0}=\mathbf{p}^{k-1}, \mathbf{z}^{k, 0}=\mathbf{z}^{k-1}$;

- For $l=0,1,2, \ldots, L-1$ : compute $\mathbf{u}^{k, l+1}$ from (43) for $\mathbf{p}=\mathbf{p}^{k, l}, \mathbf{z}=\mathbf{z}^{k, l}$; then compute $\mathbf{p}^{k, l+1}$ from (46) for $\mathbf{u}=\mathbf{u}^{k, l+1}, \mathbf{z}=\mathbf{z}^{k, l}$; and then compute $\mathbf{z}^{k, l+1}$ from (49) for $\mathbf{u}=\mathbf{u}^{k, l+1}, \mathbf{p}=\mathbf{p}^{k, l+1}$;

- $\mathbf{u}^{k}=\mathbf{u}^{k, L}, \mathbf{p}^{k}=\mathbf{p}^{k, L}, \mathbf{z}^{k}=\mathbf{z}^{k, L}$.

By the zero initialization of Algorithm 5 and induction, it is guaranteed that $\mathbf{u}^{k} \in$ $\mathbf{V}_{M}, \mathbf{p}^{k} \in \mathbf{Q}_{M}, \mathbf{z}^{k} \in \mathbf{V}_{M}, \forall k$.

Remark The problem (10) is non-convex and it is usually difficult to find its global minima. One may consider to apply an alternative minimization procedure with respect to $\mathbf{u}$ (with fixed $\mu$ ) and $\mu$ (with fixed $\mathbf{u}$ ). For fixed $\mu$, Algorithm 5 can be applied to find $\mathbf{u}$. For fixed $\mathbf{u}, \mu$ can be easily obtained by its first order optimality condition. However, this alternative minimization algorithm is not guaranteed to converge, since the problem (10) is non-convex. Currently our tests for (10) show correct segmentation results for only the case of two-region segmentation. 
Table 1 Mesh surfaces used in this paper and their sizes (numbers of vertices and triangles)

\begin{tabular}{llr}
\hline Mesh surface & \# vertices & \# triangles \\
\hline Sphere & 40962 & 81920 \\
Horse & 48485 & 96966 \\
Cameraman & 66049 & 131072 \\
Lena & 65536 & 130050 \\
Bunny & 34817 & 69630 \\
\hline
\end{tabular}

\section{Numerical Examples}

\subsection{Test Platform, Stopping Condition, and the Computation of SNR}

We implemented the proposed algorithms in $\mathrm{C}++$. All of the numerical experiments were made on a laptop with Intel Core 2 Duo $2.80 \mathrm{GHz}$ CPU and 4 GB RAM and MS Windows XP Professional x64 Edition.

In our experiments, we applied the following stopping condition:

$$
\left\|u^{k}-u^{k-1}\right\|_{V_{M}}<\epsilon \quad\left(\text { or }\left\|\mathbf{u}^{k}-\mathbf{u}^{k-1}\right\|_{\mathbf{v}_{M}}<\epsilon\right), \quad \text { for a predescribed } \epsilon>0 .
$$

See Sect. 3 for the definition of \|\|$_{V_{M}}$ and \|\|$_{\mathbf{v}_{M}}$.

The SNRs (signal-to-noise ratios) given in the examples were computed as follows. For gray images,

$$
\mathrm{SNR}=10 \log \frac{\|u-\bar{u}\|_{V_{M}}^{2}}{\|u-g\|_{V_{M}}^{2}},
$$

where $u$ is the noise-free (clean) image; $g$ is the observed or recovered image; $\bar{u}$ is the average intensity of the image $u$ on $M$. The formulae for color images is similarly defined by using the definition of the norm \|\| $\mathbf{v}_{M}$.

\subsection{Examples}

Here we give some examples. In particular, we used the following triangular mesh surfaces in our experiments; see Table 1 where the mesh sizes are shown.

A group of examples of ALM applied to TV and vectorial TV based restoration and segmentation of gray and color images are shown in Figs. 1, 2, 3, 4, 5, 6, and 7. The experimental information of these examples is given in Tables 2, 3, 4, 5, 6, 7, and 8, respectively. As one can see, our algorithms are very efficient for both gray and color image restoration and segmentation.

In image restoration, our algorithms generate reconstruction results with greatly improved SNRs in seconds. In all these restoration examples, the parameter $r$ of ALM and the stopping condition $\epsilon$ were simply set to 0.01 and $1 . e^{-7}$, respectively.

In multi-region segmentation, our algorithms also perform efficiently and generate correct segmentation results. Experiments show that they are robust to image noise and inaccurate inputs of intensity means of segments $\mu$ present in the segmentation model. The robustness to noise makes our method work for noisy images. The robustness to inaccurate inputs is of great importance in real applications, since in noisy images the intensity means $\mu$ are difficult to estimate precisely. In these segmentation examples we used uniform parameters and stopping condition; see Tables 7 and 8. 

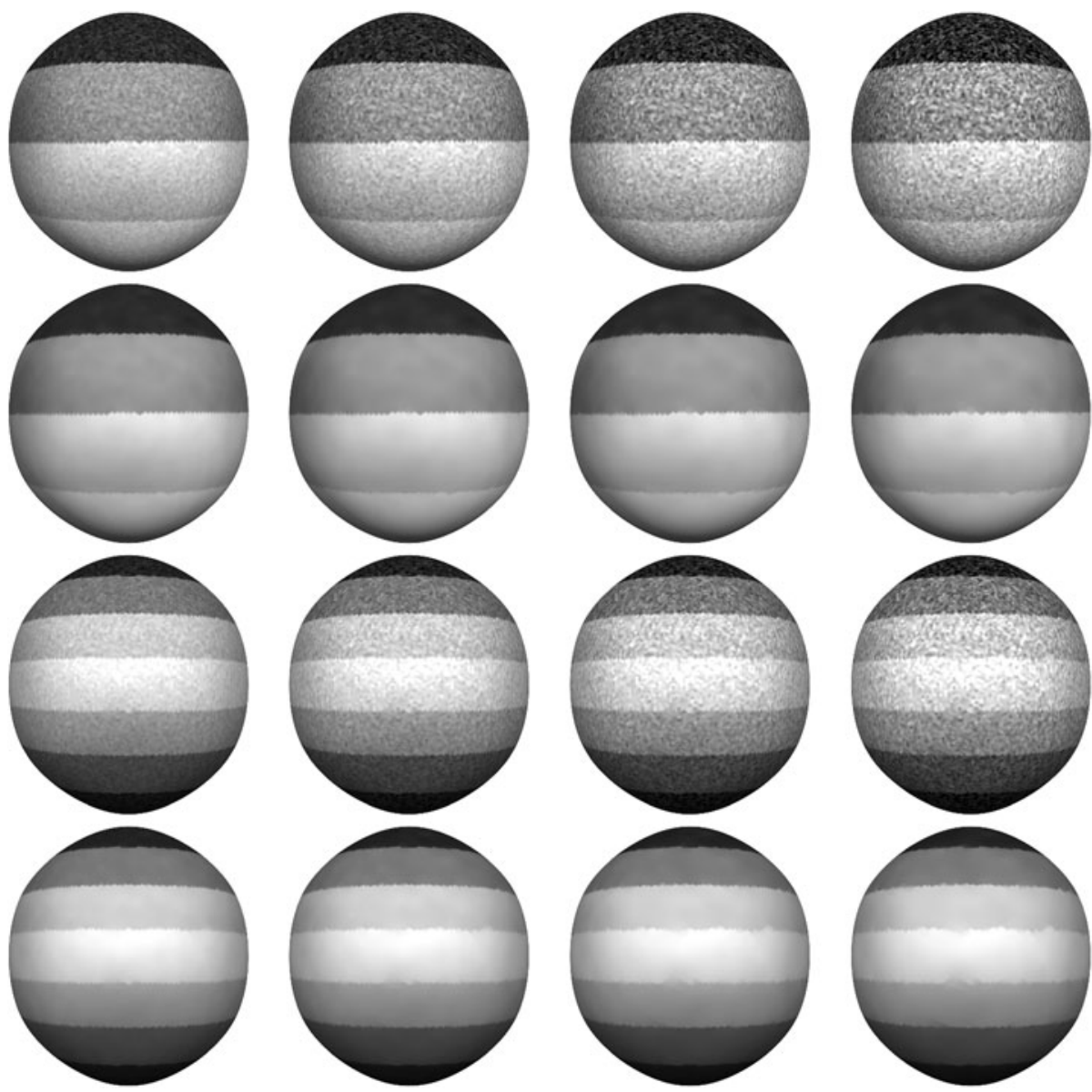

Fig. 1 Denoising two images (with non-symmetric and symmetric structures, respectively) with different levels of noise on the sphere surface based on TV restoration. The first and third rows are noisy images. From left to right, the standard deviations of the noise are $0.04,0.06,0.08,0.1$, respectively. The second and fourth rows are recovered images. See Table 2

Table 2 Experimental information about the examples in Fig. 1. The stopping condition is $\epsilon=1 . e^{-7}$

\begin{tabular}{|c|c|c|c|c|c|c|}
\hline \multirow[b]{2}{*}{ Example \# } & \multicolumn{2}{|l|}{ Observation } & \multicolumn{2}{|c|}{ Parameters } & \multicolumn{2}{|l|}{ Recovered } \\
\hline & Noise level & $\mathrm{SNR}(\mathrm{dB})$ & $\alpha$ & $r$ & $\mathrm{SNR}(\mathrm{dB})$ & $\mathrm{CPU}(\mathrm{s})$ \\
\hline Column 1 NonSymm & 0.04 & 17.5309 & 4500 & 0.01 & 30.0471 & 3.235 \\
\hline Column 2 NonSymm & 0.06 & 14.0091 & 3000 & 0.01 & 27.8715 & 3.282 \\
\hline Column 3 NonSymm & 0.08 & 11.5103 & 2500 & 0.01 & 26.2949 & 3.641 \\
\hline Column 4 NonSymm & 0.10 & 9.5721 & 2000 & 0.01 & 24.9920 & 3.781 \\
\hline Column 1 Symm & 0.04 & 17.1230 & 5000 & 0.01 & 27.9418 & 3.375 \\
\hline Column 2 Symm & 0.06 & 13.6012 & 3500 & 0.01 & 25.5181 & 3.437 \\
\hline Column 3 Symm & 0.08 & 11.1024 & 2500 & 0.01 & 23.7845 & 3.656 \\
\hline Column 4 Symm & 0.10 & 9.1642 & 2000 & 0.01 & 22.4252 & 3.484 \\
\hline
\end{tabular}



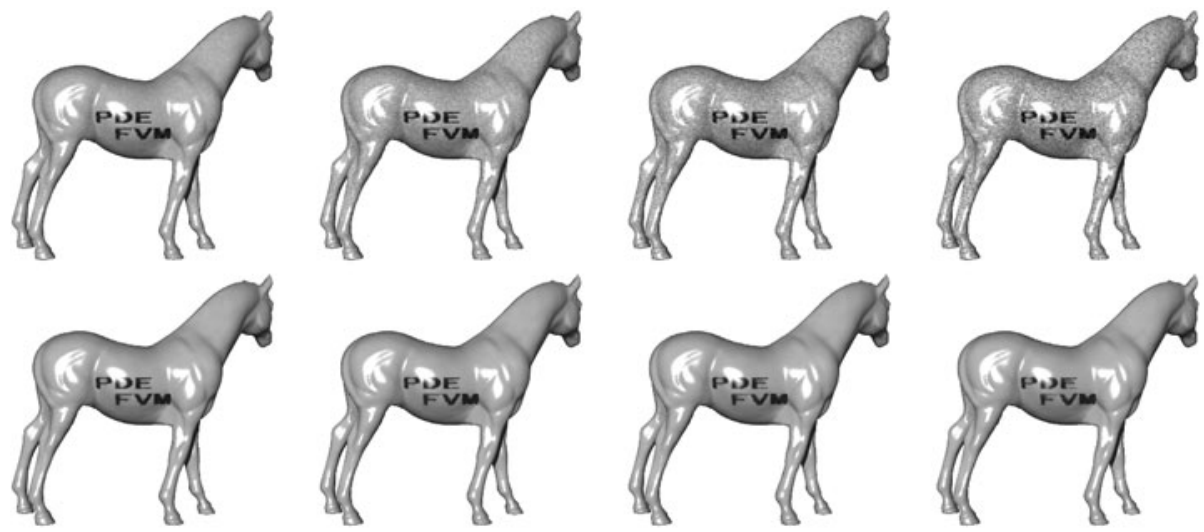

Fig. 2 Denoising a text-image with different levels of noise on the horse surface based on TV restoration. The first row are noisy images. From left to right, the standard deviations of the noise are 0.02, 0.04, 0.06, 0.08 , respectively. The second row are recovered images. See Table 3
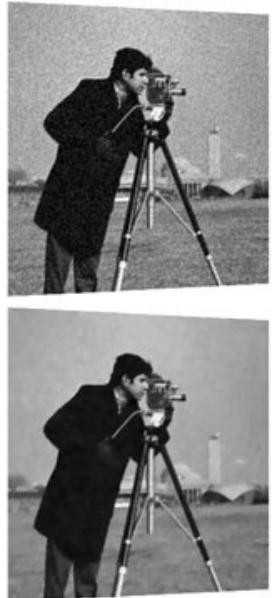
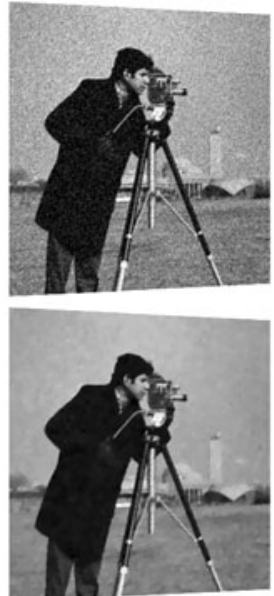
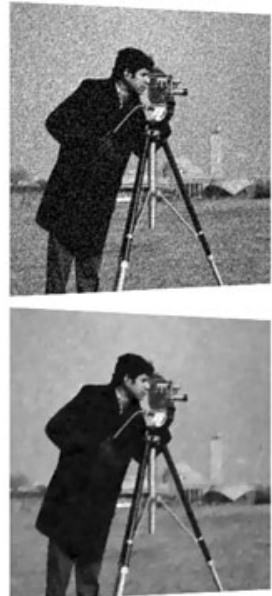
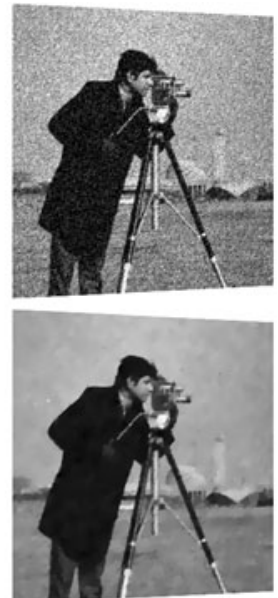

Fig. 3 Denoising the cameraman image with different levels of noise on a flat surface based on TV restoration. The first row are noisy images. From left to right, the standard deviations of the noise are $0.06,0.08$, $0.10,0.12$, respectively. The second row are recovered images. See Table 4

Table 3 Experimental information about the examples in Fig. 2. The stopping condition is $\epsilon=1 . e^{-7}$

\begin{tabular}{|c|c|c|c|c|c|c|}
\hline \multirow[b]{2}{*}{ Example \# } & \multicolumn{2}{|l|}{ Observation } & \multicolumn{2}{|c|}{ Parameters } & \multicolumn{2}{|l|}{ Recovered } \\
\hline & Noise level & $\mathrm{SNR}(\mathrm{dB})$ & $\alpha$ & $r$ & $\mathrm{SNR}(\mathrm{dB})$ & $\mathrm{CPU}(\mathrm{s})$ \\
\hline Column 1 & 0.02 & 5.8059 & 11000 & 0.01 & 16.5931 & 1.672 \\
\hline Column 2 & 0.04 & -0.2147 & 7000 & 0.01 & 13.0695 & 1.953 \\
\hline Column 3 & 0.06 & -3.7366 & 5000 & 0.01 & 10.9710 & 2.172 \\
\hline Column 4 & 0.08 & -6.2353 & 4000 & 0.01 & 9.3320 & 2.359 \\
\hline
\end{tabular}



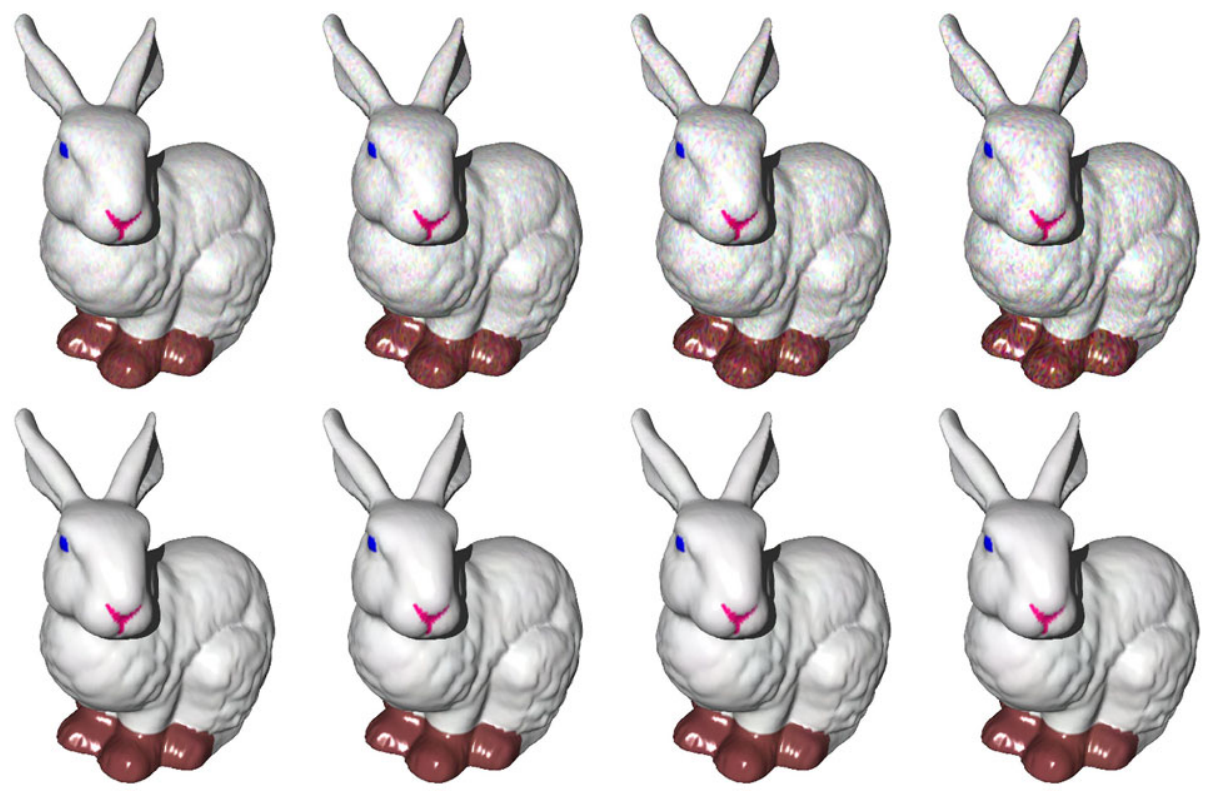

Fig. 4 Denoising a color image with different levels of noise on the Bunny surface based on vectorial TV restoration. The first row are noisy images. From left to right, the standard deviations of the noise are 0.04 , 0.06, 0.08, 0.10, respectively. The second row are recovered images. See Table 5

Table 4 Experimental information about the examples in Fig. 3. The stopping condition is $\epsilon=1 . e^{-7}$

\begin{tabular}{|c|c|c|c|c|c|c|}
\hline \multirow[b]{2}{*}{ Example \# } & \multicolumn{2}{|l|}{ Observation } & \multicolumn{2}{|c|}{ Parameters } & \multicolumn{2}{|l|}{ Recovered } \\
\hline & Noise level & $\overline{S N R}(\mathrm{~dB})$ & $\bar{\alpha}$ & $r$ & $\mathrm{SNR}(\mathrm{dB})$ & $\overline{\mathrm{CPU}(\mathrm{s})}$ \\
\hline Column 1 & 0.06 & 12.0609 & 10000 & 0.01 & 19.1417 & 3.735 \\
\hline Column 2 & 0.08 & 9.5621 & 8000 & 0.01 & 17.6617 & 3.828 \\
\hline Column 3 & 0.10 & 7.6239 & 6000 & 0.01 & 16.5206 & 3.796 \\
\hline Column 4 & 0.12 & 6.0384 & 4500 & 0.01 & 15.5818 & 4.25 \\
\hline
\end{tabular}

Table 5 Experimental information about the examples in Fig. 4. The stopping condition is $\epsilon=1 . e^{-7}$

\begin{tabular}{|c|c|c|c|c|c|c|}
\hline \multirow[b]{2}{*}{ Example \# } & \multicolumn{2}{|l|}{ Observation } & \multicolumn{2}{|c|}{ Parameters } & \multicolumn{2}{|l|}{ Recovered } \\
\hline & Noise level & $\operatorname{SNR}(\mathrm{dB})$ & $\bar{\alpha}$ & $r$ & $\mathrm{SNR}(\mathrm{dB})$ & $\overline{\mathrm{CPU}(\mathrm{s})}$ \\
\hline Column 1 & 0.04 & 10.9407 & 1500 & 0.01 & 29.5013 & 5.797 \\
\hline Column 2 & 0.06 & 7.4189 & 1200 & 0.01 & 27.5986 & 6.187 \\
\hline Column 3 & 0.08 & 4.9201 & 1000 & 0.01 & 26.2133 & 7.109 \\
\hline Column 4 & 0.10 & 2.9819 & 900 & 0.01 & 25.3085 & 8.500 \\
\hline
\end{tabular}

At the end of this section, we remark on the choice of the positive parameters $r, r_{\mathbf{p}}, r_{\mathbf{z}}$ in the augmented Lagrangian method. According to our experiments, there seem some ranges for the values of these parameters to make the algorithms stable and efficient. The algorithms 

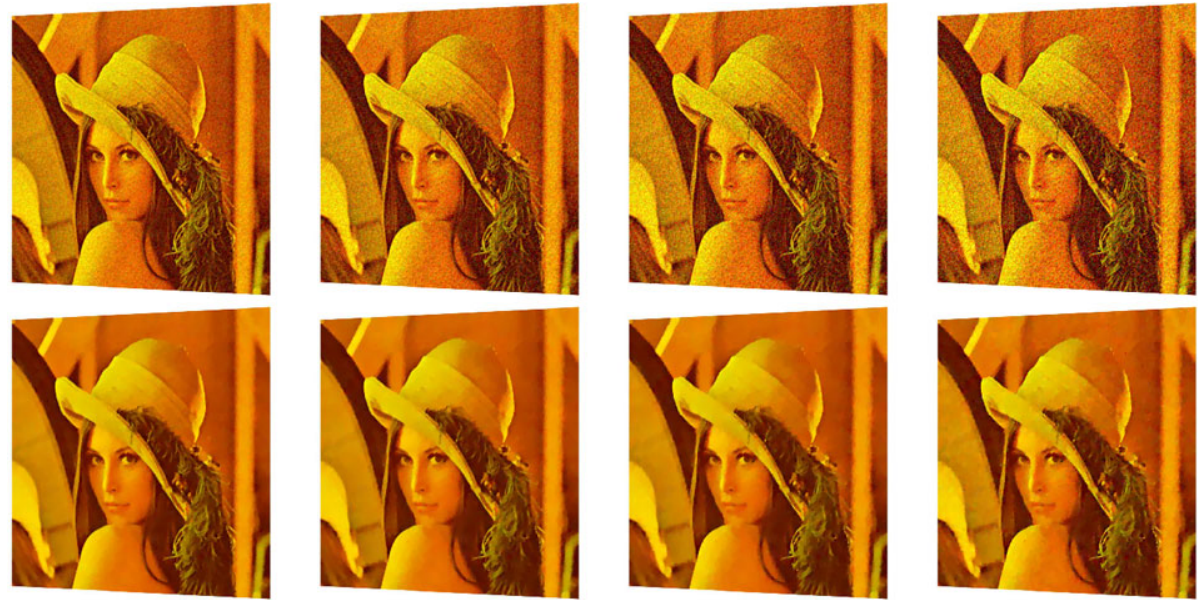

Fig. 5 Denoising the Lena (color) image with different levels of noise on a flat surface based on vectorial TV restoration. The first row are noisy images. From left to right, the standard deviations of the noise are $0.06,0.08,0.10,0.12$, respectively. The second row are recovered images. See Table 6

Table 6 Experimental information about the examples in Fig. 5. The stopping condition is $\epsilon=1 . e^{-7}$

\begin{tabular}{|c|c|c|c|c|c|c|}
\hline \multirow[b]{2}{*}{ Example \# } & \multicolumn{2}{|l|}{ Observation } & \multicolumn{2}{|c|}{ Parameters } & \multicolumn{2}{|l|}{ Recovered } \\
\hline & Noise level & $\mathrm{SNR}(\mathrm{dB})$ & $\bar{\alpha}$ & $r$ & $\mathrm{SNR}(\mathrm{dB})$ & $\mathrm{CPU}(\mathrm{s})$ \\
\hline Column 1 & 0.06 & 8.7710 & 7000 & 0.01 & 15.7636 & 8.140 \\
\hline Column 2 & 0.08 & 6.2722 & 5000 & 0.01 & 14.3979 & 9.000 \\
\hline Column 3 & 0.10 & 4.3340 & 4000 & 0.01 & 13.3181 & 9.391 \\
\hline Column 4 & 0.12 & 2.7503 & 4000 & 0.01 & 11.8496 & 9.484 \\
\hline
\end{tabular}

may be not numerically stable for too large or too small positive parameters. One potential reason is that, due to the irregularity of the mesh surface, the condition number of the coefficient matrix of the $u$-sub or $\mathbf{u}$-sub problem and hence the stability of the algorithms are more sensitive to the parameters $r, r_{\mathbf{p}}, r_{\mathbf{z}}$, compared to the case of 2D planar image processing. Consequently, $r$ (or $r_{\mathbf{p}}, r_{\mathbf{z}}$ ) depends on the model parameter $\alpha$ (or $\beta$ ) and the gradient and divergence operators. The model parameter $\alpha$ (or $\beta$ ) depends on the image to be processed. The two differential operators are determined by the structure and distribution of the triangles on the mesh surface as well as the mesh resolution, i.e., the geometry of the mesh surface. To precisely describe the relation between good parameters $r$ (or $r_{\mathbf{p}}, r_{\mathbf{z}}$ ) and $\alpha$ (or $\beta$ ) as well as the geometry of the mesh surface is quite difficult. Therefore, it is hard to obtain optimal values of $r, r_{\mathbf{p}}, r_{\mathbf{z}}$. Fortunately, our tests show that, the effective ranges of $r, r_{\mathbf{p}}, r_{\mathbf{z}}$ are large enough so that common parameters $r$ (or $r_{\mathbf{p}}, r_{\mathbf{z}}$ ) exist for different mesh surfaces and different model parameters $\alpha$ (or $\beta$ ); see the examples. Note that the effective ranges of $r$ and $r_{\mathbf{p}}, r_{\mathbf{z}}$ may be quite different even for the same mesh surface and image because they are used in different minimization problems (with different objective functions) in different applications. 

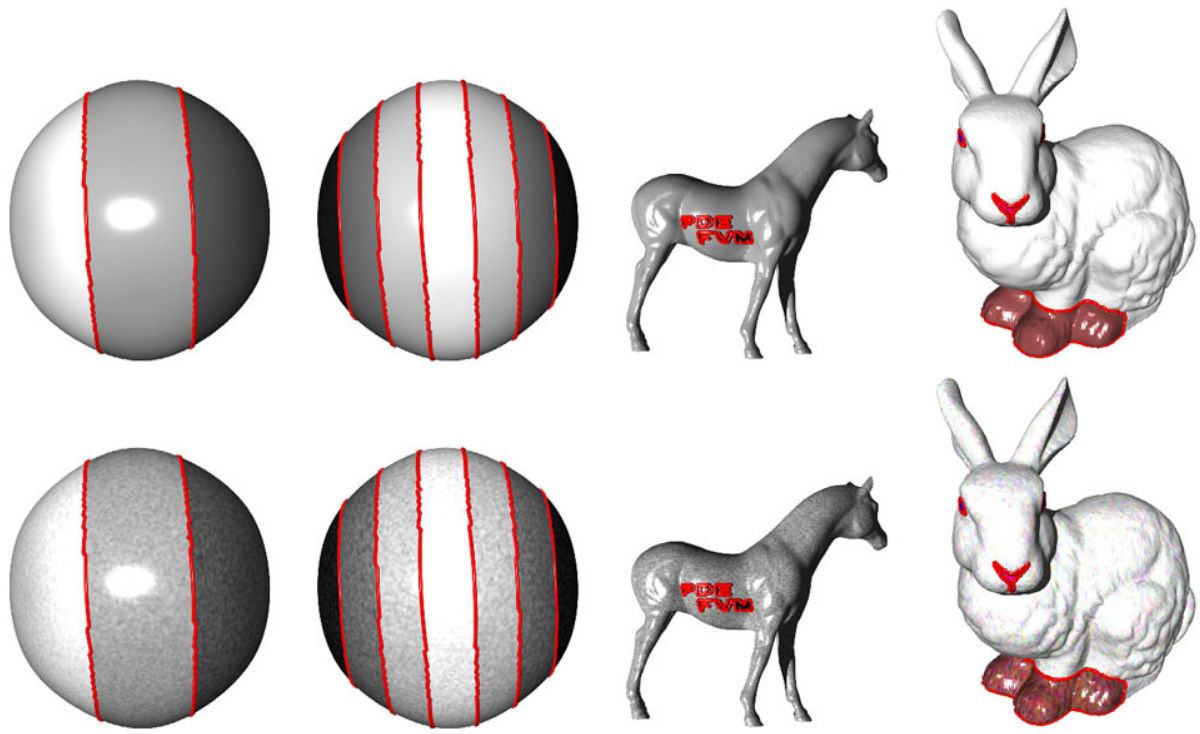

Fig. 6 Multi-region segmentation of gray and color images based on vectorial TV regularization. From left to right: the images are divided into 3,4,2, 4 segments (note that one segment may have several different disconnected domains whose intensity values are the same or similar), respectively. The first row is segmentation results on clean images, while the second row contains segmentation results on noisy images. These examples show the robustness to noise of our algorithms. See Table 7
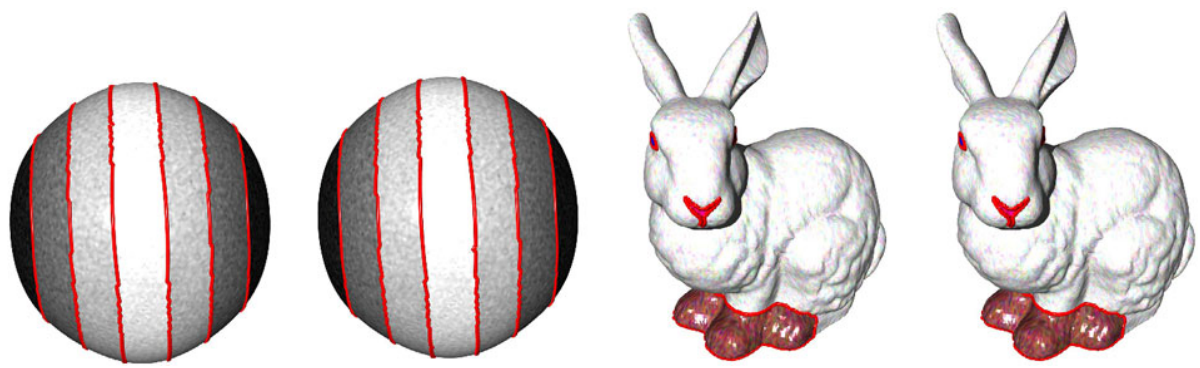

Fig. 7 Multi-region segmentation of noisy gray and color images based on vectorial TV regularization with accurate and inaccurate inputs of intensity means of segments. The input intensity means of segments in Column 1 and 3 are accurate, while those in Column 2 and 4 are inaccurate. These examples show that our algorithms still work even for inaccurate inputs of intensity means of segments. This robustness to inaccurate inputs is of importance for segmentation of noisy images, since in noisy images the intensity means of segments are difficult to estimate precisely. See Table 8

\section{Conclusion}

In this paper, we presented to use the augmented Lagrangian method to solve TV and vectorial TV based image restoration and multi-region segmentation problems on triangular mesh surfaces, based on defining inner product spaces on mesh surfaces. The provided algorithms work well for both gray and color images. Due to the use of fast solvers for linear systems and closed form solutions to subproblems, the method is extremely efficient, as demonstrated by our numerical experiments. One future work is the stability and con- 
Table 7 Experimental information about the examples in Fig. 6. In all these examples, the parameters $\left(\beta, r_{\mathbf{p}}, r_{\mathbf{z}}\right)=\left(5 . e^{-5}, 5 . e^{-8}, 1\right)$. The stopping condition is $\epsilon=1 . e^{-5}$

\begin{tabular}{llllr}
\hline Example \# & & SNR $(\mathrm{dB})$ & Intensity means of segments & CPU(s) \\
\hline Column 1 & Row 1 & $\infty$ & $(0.60 .60 .6) ;(0.30 .30 .3) ;$ & 7.485 \\
& Row 2 & 16.8889 & $(0.90 .90 .9)$ & 8.953 \\
Column 2 & Row 1 & $\infty$ & $(0.10 .10 .1) ;(0.40 .40 .4) ;$ & 16.562 \\
& Row 2 & 16.1223 & $(0.70 .70 .7) ;(0.90 .90 .9)$ & 19.359 \\
Column 3 & Row 1 & $\infty$ & $(0.5650 .5650 .565) ;(0.10 .10 .1)$ & 3.39 \\
& Row 2 & -4.40196 & & 3.937 \\
Column 4 & Row 1 & $\infty$ & $(0.90 .90 .9) ;(0.5019610 .250980 .25098) ;$ & 3.922 \\
& Row 2 & 4.92012 & $(100.501961) ;(001)$ & 4.937 \\
\hline
\end{tabular}

Table 8 Experimental information about the examples in Fig. 7. In all these examples, the parameters $\left(\beta, r_{\mathbf{p}}, r_{\mathbf{z}}\right)=\left(5 . e^{-5}, 5 \cdot e^{-8}, 1\right)$. The stopping condition is $\epsilon=1 . e^{-5}$

\begin{tabular}{|c|c|c|c|}
\hline Example \# & $\mathrm{SNR}(\mathrm{dB})$ & Intensity means of segments & $\mathrm{CPU}(\mathrm{s})$ \\
\hline Column 1 & 16.1223 & $\begin{array}{l}\left(\begin{array}{lll}0.1 & 0.1 & 0.1\end{array}\right) ;\left(\begin{array}{lll}0.4 & 0.4 & 0.4\end{array}\right) \\
\left(\begin{array}{llll}0.7 & 0.7 & 0.7\end{array}\right) ;\left(\begin{array}{lll}0.9 & 0.9 & 0.9\end{array}\right)\end{array}$ & 19.359 \\
\hline Column 2 & & 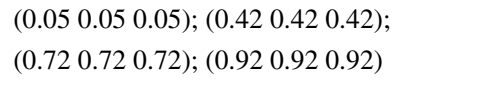 & 20 \\
\hline Column 3 & 4.92012 & $\begin{array}{l}\left(\begin{array}{lll}0.9 & 0.9 & 0.9\end{array}\right) ;\left(\begin{array}{lll}0.501961 & 0.25098 & 0.25098\end{array}\right) ; \\
\left(\begin{array}{lll}1 & 0 & 0.501961\end{array}\right) ;\left(\begin{array}{lll}0 & 0 & 1\end{array}\right)\end{array}$ & 4.937 \\
\hline Column 4 & & $\begin{array}{l}\left(\begin{array}{lll}0.55 & 0.3 & 0.3\end{array}\right) \\
\left(\begin{array}{lll}1 & 0 & 0.6\end{array}\right) ;\left(\begin{array}{lll}0.1 & 0 & 1\end{array}\right) ;\left(\begin{array}{lll}1 & 1 & 1\end{array}\right)\end{array}$ & 3.812 \\
\hline
\end{tabular}

vergence analysis of the proposed algorithms. In addition, comparisons between the augmented Lagrangian method with other optimization techniques such as iterative thresholding, Nesterov-based schemes, and primal-dual methods applied to our problems need to be investigated. To completely solve the multi-region segmentation problem in the case where the intensity means of segments are unknown is also worthy of future research. Some special techniques may be introduced to ensure global minimization of the non-convex objective functional.

\section{References}

1. Rudin, L., Osher, S., Fatemi, E.: Nonlinear total variation based noise removal algorithms. Physica D 60, 259-268 (1992)

2. Sapiro, G., Ringach, D.: Anisotropic diffusion of multivalued images with applications to color filtering. IEEE Trans. Image Process. 5(11), 1582-1586 (1996)

3. Blomgren, P., Chan, T.: Color tv: total variation methods for restoration of vector-valued images. IEEE Trans. Image Process. 7(3), 304-309 (1998)

4. Chan, T., Kang, S., Shen, J.: Total variation denoising and enhancement of color images based on the CB and HSV color models. J. Vis. Commun. Image Rep. 12, 422-435 (2001) 
5. Bresson, X., Chan, T.: Fast dual minimization of the vectorial total variation norm and applications to color image processing. Inverse Problems and Imaging 2(4), 455-484 (2008)

6. Chan, T., Golub, G., Mulet, P.: A nonlinear primal-dual method for total variation-based image restoration. SIAM J. Sci. Comput. 20, 1964-1977 (1999)

7. Carter, J.: Dual methods for total variation based image restoration. Ph.D. thesis (2001)

8. Chambolle, A.: An algorithm for total variation minimization and applications. J. Math. Imaging Vision 20, 89-97 (2004)

9. Zhu, M., Wright, S., Chan, T.: Duality-based algorithms for total variation image restoration. Tech. Rep. 08-33 (2008)

10. Wang, Y., Yang, J., Yin, W., Zhang, Y.: A new alternating minimization algorithm for total variation image reconstruction. SIAM J. Imaging Sci. 1, 248-272 (2008)

11. Yang, J., Yin, W., Zhang, Y., Wang, Y.: A fast algorithm for edge-preserving variational multichannel image restoration. Tech. Rep. 08-50 (2008)

12. Huang, Y., Ng, M., Wen, Y.: A fast total variation minimization method for image restoration. SIAM Multiscale Model. Simul. 7, 774-795 (2009)

13. Yin, W., Osher, S., Goldfarb, D., Darbon, J.: Bregman iterative algorithms for compressend sensing and related problems. SIAM J. Imaging Sci. 1, 143-168 (2008)

14. Goldstein, T., Osher, S.: The split bregman method for 11 regularized problems. SIAM J. Imaging Sci. 2, 323-343 (2009)

15. Tai, X.C., Wu, C.: Augmented lagrangian method, dual methods and split bregman iteration for rof model. In: Proc. Scale Space and Variational Methods in Computer Vision, Second International Conference (SSVM) 2009, pp. 502-513 (2009)

16. Weiss, P., Blanc-Fraud, L., Aubert, G.: Efficient schemes for total variation minimization under constraints in image processing. SIAM J. Sci. Comput. 31, 2047-2080 (2009)

17. Wu, C., Tai, X.C.: Augmented lagrangian method dual methods, and split bregman iteration for rof, vectorial tv, and high order models. SIAM J. Imaging Sci. 3(3), 300-339 (2010)

18. Wu, C., Zhang, J., Tai, X.C.: Augmented lagrangian method for total variation restoration with nonquadratic fidelity. Inverse Problems and Imaging 5(1), 237-261 (2011)

19. Zhang, X., Burger, M., Osher, S.: A unified primal-dual algorithm framework based on bregman iteration. J. Sci. Comput. 46, 20-46 (2011)

20. Michailovich, O.: An iterative shrinkage approach to total-variation image restoration. IEEE Trans. Image Process. (2011)

21. Beck, A., Teboulle, M.: A fast iterative shrinkage-thresholding algorithm for linear inverse problems. SIAM J. Imaging Sci. 2, 183-202 (2009)

22. Chan, R., Chan, T., Shen, L., Shen, Z.: Wavelet algorithms for high-resolution image reconstruction. SIAM J. Sci. Comput. 24, 1408-1432 (2003)

23. Daubechies, I., Defrise, M., Mol, C.D.: An iterative thresholding algorithm for linear inverse problems with a sparsity constraint. Comm. Pure and Appl. Math. 57, 1413-1457 (2004)

24. Figueiredo, M., Nowak, R.: An em algorithm for wavelet-based image restoration. IEEE Trans. Image Process. 12, 906-916 (2003)

25. Kass, M., Witkin, A., Terzopoulos, D.: Snakes: active contour models. Int'1. J. Comput. Vis. 1, 321-331 (1988)

26. Caselles, V., Kimmel, R., Sapiro, G.: Geodesic active contours. Int'1 J. Comput. Vision 22, 61-79 (1997)

27. Goldenberg, R., Kimmel, R., Rivlin, E., Rudzsky, M.: Fast geodesic active contours. IEEE Trans. Image Process. 10, 1467-1475 (2001)

28. Chan, T., Vese, L.: Active contours without edges. IEEE Trans. Image Process. 10(2), 266-277 (2001)

29. Osher, S., Fedkiw, R.: Level Set Methods and Dynamic Implicit Surfaces. Springer, Berlin (2002)

30. Lie, J., Lysaker, M., Tai, X.: A variant of the level set method and applications in image segmentation. Math. Comp. 75, 1155-1174 (2006)

31. Vese, L., Chan, T.: A multiphase level set framework for image segmentation using the mumford-shah model. Int'1 J. Comput. Vision 50(3), 271-293 (2002)

32. Cremers, D., Tischhauser, F., Weickert, J., Schnorr, C.: Diffusion snakes: introducing statistical shape knowledge into the mumfordëshah functional. Int'1 J. Computer Vision 50(3), 295-313 (2002)

33. Lie, J., Lysaker, M., Tai, X.: A binary level set model and some applications to mumford-shah image segmentation. IEEE Trans. Image Process. 15, 1171-1181 (2006)

34. Mumford, D., Shah, J.: Optimal approximations by piecewise smooth functions and associated variational problems. Comm. Pure Appl. Math. 42, 577-685 (1989)

35. Appleton, B., Talbot, H.: Globally minimal surfaces by continuous maximal flows. IEEE Trans. Pattern Anal. Mach. Intell. (1) 28, 106-118 (2006)

36. Nikolova, M., Esedoglu, S., Chan, T.: Algorithms for finding global minimizers of image segmentation and denoising models. SIAM J. Appl. Math. 66(5), 1632-1648 (2006) 
37. Bresson, X., Esedoglu, S., Vandergheynst, P., Thiran, J., Osher, S.: Fast global minimization of the active contour/snake model. J. Math. Imaging Vision 28(2), 151-167 (2007)

38. Zach, C., Gallup, D., Frahm, J.M., Niethammer, M.: Fast global labeling for real-time stereo using multiple plane sweeps. In: Proc. Vision, Modeling and Visualization Workshop (VMV) (2008)

39. Pock, T., Schoenemann, T., Graber, G., Bischof, H., Cremers, D.: A convex formulation of continuous multi-label problems. In: Proc. European Conference on Computer Vision (ECCV 2008), pp. III, pp. 792-805 (2008)

40. Pock, T., Cremers, D., Bischof, H., Chambolle, A.: An algorithm for minimizing the mumford-shah functional. In: Proc. ICCV (2009)

41. Pock, T., Chambolle, A., Cremers, D., Bischof, H.: A convex relaxation approach for computing minimal partitions. In: Proc. CVPR (2009)

42. Lellmann, J., Kappes, J., Yuan, J., Becker, F., Schnörr, C.: Convex multi-class image labeling by simplexconstrained total variation. In: Proc. Second International Conference on Scale Space and Variational Methods in Computer Vision (SSVM 2009), pp. 150-162. Springer, Berlin (2009)

43. Bae, E., Yuan, J., Tai, X.: Global minimization for continuous multiphase partitioning problems using a dual approach. Tech. rep. (2009). URL: ftp://ftp.math.ucla.edu/pub/camreport/cam09-75.pdf

44. Brown, E., Chan, T., Bresson, X.: A convex approach for multi-phase piecewise constant mumford-shah image segmentation. Tech. rep. (2009). URL: ftp://ftp.math.ucla.edu/pub/camreport/cam09-66.pdf

45. Goldstein, T., Bresson, X., Osher, S.: Geometric applications of the split bregman method: segmentation and surface reconstruction. J. Sci. Comput. 45, 272-293 (2010)

46. Brown, E., Chan, T., Bresson, X.: A convex relaxation method for a class of vector-valued minimization problems with applications to mumford-shah segmentation. Tech. rep. (2010). URL: ftp://ftp.math.ucla.edu/pub/camreport/cam10-43.pdf

47. Brown, E., Chan, T., Bresson, X.: Globally convex chan-vese image segmentation. Tech. rep. (2010). URL: ftp://ftp.math.ucla.edu/pub/camreport/cam10-44.pdf

48. Lellmann, J., Becker, F., Schnörr, C.: Convex optimization for multi-class image labeling with a novel family of total variation based regularizers. In: Proc. IEEE International Conference on Computer Vision (ICCV), pp. 646-653 (2009)

49. Lellmann, J., Schnoerr, C.: Continuous multiclass labeling approaches and algorithms. Tech. rep., Univ. of Heidelberg (2010). URL: http://www.ub.uni-heidelberg.de/archiv/10460/

50. Wu, C., Deng, J., Chen, F.: Diffusion equations over arbitrary triangulated surfaces for filtering and texture applications. IEEE Trans. Visual. Comput. Graph. 14(3), 666-679 (2008)

51. Wu, C., Deng, J., Chen, F., Tai, X.: Scale-space analysis of discrete filtering over arbitrary triangulated surfaces. SIAM J. Imaging Sci. 2(2), 670-709 (2009)

52. Delaunoy, A., Fundana, K., Prados, E., Heyden, A.: Convex multi-region segmentation on manifolds. In: Proc. 12th IEEE International Conference on Computer Vision (ICCV), pp. 662-669 (2009)

53. Lai, R., Chan, T.: A framework for intrinsic image processing on surfaces. Tech. Rep. 10-25 (2010). URL: ftp://ftp.math.ucla.edu/pub/camreport/cam10-25.pdf

54. Hirani, A.: Discrete exterior calculus. Ph.D. thesis, California Institute of Technology (2003)

55. Michelot, C.: A finite algorithm for finding the projection of a point onto the canonical simplex of $\mathrm{rn}$. J. Optim. Theory Appl. 50(1), 195-200 (1986) 